\title{
Kalman filtering for adaptive nonuniformity correction in infrared focal-plane arrays
}

\author{
Sergio N. Torres \\ Department of Electrical Engineering, University of Concepcion, Concepcion, Chile \\ Majeed M. Hayat \\ Department of Electrical and Computer Engineering, University of New Mexico, Albuquerque, \\ New Mexico 87131-1356
}

Received August 16, 2002; revised manuscript received October 24, 2002; accepted October 25, 2002

\begin{abstract}
A novel statistical approach is undertaken for the adaptive estimation of the gain and bias nonuniformity in infrared focal-plane array sensors from scene data. The gain and the bias of each detector are regarded as random state variables modeled by a discrete-time Gauss-Markov process. The proposed Gauss-Markov framework provides a mechanism for capturing the slow and random drift in the fixed-pattern noise as the operational conditions of the sensor vary in time. With a temporal stochastic model for each detector's gain and bias at hand, a Kalman filter is derived that uses scene data, comprising the detector's readout values sampled over a short period of time, to optimally update the detector's gain and bias estimates as these parameters drift. The proposed technique relies on a certain spatiotemporal diversity condition in the data, which is satisfied when all detectors see approximately the same range of temperatures within the periods between successive estimation epochs. The performance of the proposed technique is thoroughly studied, and its utility in mitigating fixed-pattern noise is demonstrated with both real infrared and simulated imagery. (C) 2003 Optical Society of America
\end{abstract}

OCIS codes: $100.2000,100.2550,110.4280,110.3080,100.3020$.

\section{INTRODUCTION}

Modern imaging systems are ubiquitous in a wide range of military and civilian applications including thermal imaging, night vision, surveillance systems, astronomy, fire detection, robotics, and spectral sensing and imaging. ${ }^{1}$ At the heart of most modern imaging systems is the focalplane array (FPA), which consists of a mosaic of detectors positioned at the focal plane of an imaging lens. However, the performance of FPAs is known to be strongly affected by the spatial nonuniformity in the photoresponse of the detectors in the array, also known as fixed-pattern noise, which becomes particularly severe in mid- to far-IR imaging systems. Despite the advances in detector technology in recent years, detector nonuniformity continues to be a serious challenge, degrading spatial resolution, radiometric accuracy, and temperature resolvability. Moreover, what makes the nonuniformity problem more challenging is the fact that spatial nonuniformity drifts slowly in time; thus a one-time factory calibration will not provide a permanent remedy to the problem.

Nonuniformity correction (NUC) techniques are categorized into two classes, namely, calibration-based and scene-based techniques. In the commonly used two-point calibration technique, ${ }^{2}$ for example, the normal operation of the FPA is halted as the camera images a uniform calibration target (typically, a blackbody radiation source) at two distinct and known temperatures. The gain and the bias of each detector are then calibrated across the array so that all detectors produce a radiometrically accurate and uniform readout at the two reference temperatures. Scene-based correction algorithms, on the other hand, do provide significant cosmetic NUC without the need to halt the camera's normal operation; however, this convenience comes at the expense of compromising radiometric accuracy. Scene-based techniques typically use an image sequence and rely on motion (or changes in the actual scene) to provide diversity in the scene temperature per detector. This temperature diversity, in turn, provides a "statistical" reference point, common to all detectors, according to which the individual detector's responses can be normalized.

In recent years, a number of scene-based NUC techniques have been reported in the literature. Narendra and Foss ${ }^{3,4}$ and, more recently, Harris and Chiang ${ }^{5-7}$ developed algorithms that continually compensate for bias and gain nonuniformity by using the constant-statistics assumption. This assumption postulates that, in time, the mean and the standard deviation of the irradiance flux become the same for every detector. Under this assumption and by employing a linear model for the detector response, they showed that the mean and the standard deviation of each detector's readout signal can be regarded as its bias and gain, respectively. Scribner et $a l .{ }^{8}$ proposed a least-mean-square-error technique that resembles adaptive temporal high-pass filtering. By adjusting the time constant of the filter, their algorithm was used to reduce the spatial noise caused by bias nonuniformity (the gain correction was performed separately). A neural-network implementation of the adaptive leastmean-square-error algorithm was also developed by Scribner et al. ${ }^{9,10}$ O'Neil, ${ }^{11}$ Hardie et al., ${ }^{12}$ and Hepfer et $a l .{ }^{13}$ developed NUC techniques that rely on the fact 
that detectors that record the same scene point at different times should have the same response. For example, O'Neil uses frames of data produced by dithering the detector line of sight between consecutive frames in a known pattern. In contrast, the technique developed by Hardie et al. does not assume deterministic motion but instead uses a motion-estimation algorithm to trace the true scene value at a particular location and frame along a motion trajectory of pixels. Hayat et al. ${ }^{14}$ developed a statistical algorithm that relies on a key assumption that, in time, all detectors in the array are exposed to the same range of irradiance, which is further modeled by a uniformly distributed random variable with a constant range. Recently, Ratliff et al. ${ }^{15}$ developed an algebraic (nonstatistical) scene-based NUC technique that does not rely on any statistical or scene-diversity assumptions about the scene temperature. The algorithm utilizes estimates of interframe subpixel motion and a linear interpolation model for image motion to unify the biases of the detectors.

A key limitation of all the scene-based NUC techniques published to date is that they do not exploit any temporal statistics of the drift in the nonuniformity. As a result, each time that a drift occurs, a full-scale NUC is performed, a process that may be greatly simplified and improved if statistical knowledge on the nature of drift is exploited, especially in cases where the drift is small. In this paper, we regard the gain and the bias of each detector as state variables modeled by a Gauss-Markov random process. We use this model to develop a Kalman filter that updates the estimates of the gain and the bias of each detector in the FPA. In our formulation, the input to the Kalman filter is a sequence of fixed-length vectors of detector readout values, representing a block of frames over which no significant drift occurs in the detector's gains and biases. As drift occurs and a new vector of observations arrives, the Kalman filter updates the estimates of the gain and the bias of each detector.

This paper is organized as follows. The GaussMarkov model for the gain and the bias, along with the output model, is given in Section 2 . In Section 3, the Kalman filter is derived, and its computational efficiency is discussed. In Section 4, the proposed technique is applied to simulated data, and its performance is evaluated. In Section 5, the technique is applied to real infrared (IR) data. The conclusions are given in Section 6 .

\section{MODEL}

In this paper, we adopt the commonly used linear model for the detector response. ${ }^{1}$ For each detector in the array, vectors of readout values are considered, corresponding to a series of blocks of frames for which no significant drift in the gain and the bias occurs in each block. For the $k$ th block of frames, the output of the $i j$ th detector in the $n$th frame is approximated by

$$
Y_{k}^{i j}(n)=A_{k}^{i j} T_{k}^{i j}(n)+B_{k}^{i j}+V_{k}^{i j}(n),
$$

where $A_{k}^{i j}$ and $B_{k}^{i j}$ are, respectively, the gain and the bias associated with the $i j$ th detector in the $k$ th block of frames and $T_{k}^{i j}(n)$ is the average number of photons collected by the $i j$ th detector in the $n$th frame. The term
$V_{k}^{i j}(n)$ represents the additive temporal readout noise associated with the $i j$ th detector in the $n$th frame. Now, for the $i j$ th detector, the observation vector corresponding to the $k$ th block is $\mathbf{Y}_{k}^{i j}=\left[\begin{array}{llll}Y_{k}^{i j}(1) & \cdots & Y_{k}^{i j}\left(l_{k}\right)\end{array}\right]^{\prime}$, which is an array of length $l_{k}$ of readout values, where $l_{k}$ is the length of the $k$ th block of frames. For brevity of notation, the pixel index $i j$ will be omitted whenever convenient. For example, we may write $T_{k}(n), A_{k}, B_{k}$, and $\mathbf{Y}_{k}$ in place of $T_{k}^{i j}(n), A_{k}^{i j}, B_{k}^{i j}$, and $\mathbf{Y}_{k}^{i j}$, respectively.

We are ultimately interested in the recursive and minimum-mean-square-error (MMSE) estimation of the two-dimensional state vector $\mathbf{X}_{k}=\left[A_{k}, B_{k}\right]^{\prime}$ given the sequence of vector observations $\mathbf{Y}_{1}, \ldots, \mathbf{Y}_{k}$. From the orthogonality principle, it follows that the above MMSE estimate, denoted by $\hat{\mathbf{X}}_{k}=\left[\hat{A}_{k}, \hat{B}_{k}\right]^{\prime}$, must obey the relation

$$
\mathrm{E}\left[\left(\mathbf{X}_{k}-\hat{\mathbf{X}}_{k}\right) \mathbf{Y}_{l}^{\prime}\right]=0, \quad l=1, \ldots, k .
$$

Equivalently, $\hat{\mathbf{X}}_{k}$ can be regarded as the conditional expectation of $\mathbf{X}_{k}$ given $\mathbf{Y}_{1}, \ldots, \mathbf{Y}_{k}$, i.e.,

$$
\hat{\mathbf{X}}_{k}=\mathrm{E}\left[\mathbf{X}_{k} \mid \mathbf{Y}_{1}, \ldots, \mathbf{Y}_{k}\right]
$$

To obtain the above MMSE estimate in a Kalman-filtering setup, we need two mathematical models, namely, Eq. (1) the state equation model, which characterizes the dynamics of the gain and the bias (i.e., the Gauss-Markov model), and Eq. (2) the observation model, which is an extension of the model presented in Eq. (1). We now develop these two models.

\section{A. State Equations for the Gain and the Bias}

Motivated by the fact that the drift in the gain and the bias occurs slowly in time (i.e., from one block of frames to another), we wish to think of the gain and the bias at the $(k+1)$ th block as a random perturbation of the gain and the bias in the $k$ th block. In the context of GaussMarkov processes, we may express this perturbation model by representing the state vector $\mathbf{X}_{k}$ with the following autoregressive model:

$$
\mathbf{X}_{k+1}=\mathbf{\Phi}_{k} \mathbf{X}_{k}+\mathbf{W}_{k},
$$

where

$$
\boldsymbol{\Phi}_{k}=\left[\begin{array}{cc}
\alpha_{k} & 0 \\
0 & \beta_{k}
\end{array}\right]
$$

is called the state transition matrix and $\mathbf{W}_{k}$ $=\left[W_{k}^{(1)}, W_{k}^{(2)}\right]^{\prime}$ contains the driver noise sources for the gain and the bias, respectively. The parameters $0 \leqslant \alpha_{k}$ $<1$ and $0 \leqslant \beta_{k}<1$ are chosen according to the magnitude of the drift between times $k$ and $k+1$. The driver noise processes associated with the gain and the bias are each assumed to be white, Gaussian, and mutually uncorrelated. The cross covariance of the two-dimensional driver noise process $\mathbf{W}_{k}$ is therefore given by

$$
\mathbf{Q}_{k}=\left[\begin{array}{cc}
\sigma_{W_{k}^{(1)}}^{2} & 0 \\
0 & \sigma_{W_{k}^{(2)}}^{2}
\end{array}\right],
$$

where $\sigma_{W_{k}^{(1)}}^{2}$ and $\sigma_{W_{k}^{(2)}}^{2}$ are the variances of the driver noise for the gain and the bias, respectively. The assigned val- 
ues of these variances, however, will play a key role in the evolution of the fixed-pattern noise and must be handled delicately.

In the above model, it is important to ensure that the stochastic mechanism for the gain and bias drift does not lead to any long-term changes in the dynamic range of the detector's readout values. More precisely, although the gain and the bias are allowed to drift randomly, we should maintain that the net drift is zero on average. Thus we require that the mean of the state vector $\mathbf{X}_{k}$ be constant with respect to $k$. In particular, if we assume that $\alpha_{0}$ $=\alpha_{1}=\cdots=\alpha_{k} \triangleq \alpha$ and $\beta_{0}=\beta_{1}=\cdots=\beta_{k} \triangleq \beta$, a simple calculation shows that the stationary-mean requirement translates into the following condition:

$$
\mathbf{M}_{k} \triangleq \mathrm{E}\left[\mathbf{W}_{k}\right]^{\prime}=\overline{\mathbf{X}}_{0}^{\prime}\left[\begin{array}{cc}
1-\alpha & 0 \\
0 & 1-\beta
\end{array}\right], \quad k \geqslant 0
$$

where

$$
\overline{\mathbf{X}}_{0}=\mathrm{E}\left[\mathbf{X}_{0}\right] \triangleq\left[\bar{A}_{0}, \bar{B}_{0}\right]^{\prime} .
$$

The mean initial gain and bias, $\bar{A}_{0}$ and $\bar{B}_{0}$, are assumed to be known and common to all detectors. They can be taken as the nominal gain and bias values for the FPA provided by the manufacturer. Finally, although the drift in the gain and the bias changes the fixed-pattern noise, it should not alter its severity. Hence the GaussMarkov model given by Eq. (4) must also have a stationary variance. The variances of the driver noise sources are therefore derived under the requirement that $\mathrm{E}\left[\mathbf{X}_{k} \mathbf{X}_{k}^{\prime}\right]=\mathrm{E}\left[\mathbf{X}_{0} \mathbf{X}_{0}^{\prime}\right]$. With these requirements and by using the assumption that the gain and the bias are uncorrelated, one can show that the variances of the driver noise sources at the $k$ th observation vector time are determined in terms of the correlation parameters and the initial variances. In particular, for $k \geqslant 0$,

$$
\sigma_{W_{k}^{(1)}}^{2}=\left(1-\alpha^{2}\right) \sigma_{A_{0}}^{2}, \quad \sigma_{W_{k}^{(2)}}^{2}=\left(1-\beta^{2}\right) \sigma_{B_{0}}^{2},
$$

where $\sigma_{A_{0}}^{2}$ and $\sigma_{B_{0}}^{2}$ are the variances of the gain and the bias at $k=0$, respectively, representing the initial gain and bias in the FPA, and they are assumed to be known.

\section{B. Observation Model for a Block of Frames}

We can write the observation vector $\mathbf{Y}_{k}$ compactly as

$$
\mathbf{Y}_{k}=\mathbf{H}_{k} \mathbf{X}_{k}+\mathbf{V}_{k},
$$

where $\mathbf{H}_{k}$ denotes the observation matrix, given by

$$
\mathbf{H}_{k}=\left[\begin{array}{cc}
T_{k}(1) & 1 \\
\vdots & \vdots \\
T_{k}\left(l_{k}\right) & 1
\end{array}\right]
$$

and $\mathbf{V}_{k}=\left[\begin{array}{llll}V_{k}(1) & \cdots & V_{k}\left(l_{k}\right)\end{array}\right]^{\prime}$ is the readout noise vector for the $k$ th block.

As indicated in Section 1, we will adopt the constantrange assumption. ${ }^{14}$ (The constant-range requirement, along with its predecessor, the constant-statistics requirement, ${ }^{3-7}$ has been shown to serve well as statistical reference points providing a common baseline according to which the gain and bias nonuniformity in detectors is compensated.) Namely, for each block of frames (the $k$ th, say), we will assume that the average number of photons $T^{i j}(n)$ in the block in any detector $(i, j)$ is an independent sequence of uniformly distributed random variables in the range $\left[T_{k}^{\min }, T_{k}^{\max }\right]$, which is common to all detectors and frames within the block. Our experience indicates that the constant-range condition can be satisfied, for example, in the presence of adequate motion (global or local), as shown in the examples to come. For simplicity, it is also assumed that the observation noise term sequence $\left\{V_{i j}(n)\right\}$ is white and independent of the signal sequence $\left\{T^{i j}(n)\right\}$ with the covariance matrix

$$
\mathbf{R}_{k}=\mathbf{I}_{l_{k}} \sigma_{\mathbf{V}_{k}}^{2},
$$

where $\mathbf{I}_{l_{k}}$ is the $l_{k} \times l_{k}$ identity matrix and $\sigma_{\mathbf{V}_{k}}^{2}$ is the variance of the additive observation noise in the $k$ th block time, which is assumed to be known.

With the above stochastic dynamical model [described by Eqs. (4) and (10)] at hand, we proceed to obtain a Kalman filter to recursively (in terms of blocks of frames) estimate the gain and the bias in each detector.

\section{RECURSIVE ESTIMATION OF THE GAIN AND THE BIAS}

In this section, we present a recursive linear MMSE filter for the estimation of the system state $\mathbf{X}_{k}$ given $\mathbf{Y}_{1}, \ldots, \mathbf{Y}_{k}$. The Kalman filter is derived following the general procedure given in Refs. 16-18, which is based on the orthogonality principle (2) [or, equivalently, based on the conditional expectation given in Eq. (3)]. The derivation can be outlined in four main steps: (1) derivation of the predictor estimate of the state vector, (2) derivation of the predictor estimate of the observation vector, (3) derivation of the Kalman gain, and (4) derivation of a recursive equation for the error covariance matrix. The special structure of the observation matrix, given in Eq. (11), and the fact that it is stochastic will play an important role in steps 2 and 3 . These two steps involve performing certain nonstandard calculations, which are included in Appendix A. The calculations involved in the remaining steps are straightforward, and the details are omitted. The final results are given below.

\section{A. Kalman Filter}

For the $k$ th block $(k \geqslant 1)$ and each detector, the MMSE estimate $\hat{\mathbf{X}}_{k}$, given the data vector $\mathbf{Y}_{k}$ in the block, is computed iteratively by using the relation

$$
\hat{\mathbf{X}}_{k}=\hat{\mathbf{X}}_{k}^{-}+\mathbf{K}_{k}\left(\mathbf{Y}_{k}-\overline{\mathbf{H}}_{k} \hat{\mathbf{X}}_{k}^{-}\right)
$$

where $\hat{\mathbf{X}}_{k}^{-}$is called the predictor estimate, defined as $\hat{\mathbf{X}}_{k}^{-} \triangleq \mathrm{E}\left[\mathbf{X}_{k} \mid \mathbf{Y}_{1}, \ldots, \mathbf{Y}_{k-1}\right]$, and can be iteratively computed by using

$$
\hat{\mathbf{X}}_{k}^{-}=\boldsymbol{\Phi}_{k-1} \hat{\mathbf{X}}_{k-1}+\mathbf{M}_{k-1}^{\prime}
$$

where $\boldsymbol{\Phi}_{k-1}$ and $\mathbf{M}_{k-1}$ are given by Eqs. (5) and (7), respectively. The $\left(2 \times l_{k}\right)$ matrix $\mathbf{K}_{k}$ is termed the Kalman gain matrix and can be computed by using

$$
\mathbf{K}_{k}=\mathbf{P}_{k}^{-} \overline{\mathbf{H}}_{k}^{\prime}\left[\overline{\mathbf{H}}_{k} \mathbf{P}_{k}^{-} \overline{\mathbf{H}}_{k}^{\prime}+\mathbf{R}_{k}+\sigma_{T}^{2}\left(\sigma_{A_{0}}^{2}+\bar{A}_{0}\right) \mathbf{I}_{l_{k}}\right]^{-1},
$$


where $\mathbf{R}_{k}$ is given by Eq. (12), and $\sigma_{T}^{2}$ and the $\left(l_{k} \times 2\right)$ matrix $\overline{\mathbf{H}}_{k}$ are, respectively, the variance of the IR signal $T$ and the mean of the observation matrix, which are given by

$$
\begin{aligned}
\sigma_{T}^{2} & =\frac{1}{12}\left(T_{k}^{\min }-T_{k}^{\max }\right)^{2}, \\
\overline{\mathbf{H}}_{k} & =\left[\begin{array}{cc}
0.5\left(T_{k}^{\min }+T_{k}^{\max }\right) & 1 \\
\vdots & \vdots \\
0.5\left(T_{k}^{\min }+T_{k}^{\max }\right) & 1
\end{array}\right] .
\end{aligned}
$$

Finally, $\mathbf{P}_{k}^{-}$is termed the a priori error covariance matrix, which is defined as

$$
\mathbf{P}_{k}^{-} \triangleq \mathrm{E}\left[\left(\mathbf{X}_{k}-\hat{\mathbf{X}}_{k}^{-}\right)\left(\mathbf{X}_{k}-\hat{\mathbf{X}}_{k}^{-}\right)^{\prime}\right],
$$

and can be evaluated iteratively by employing the relation

$$
\mathbf{P}_{k}^{-}=\boldsymbol{\Phi}_{k-1} \mathbf{P}_{k-1} \boldsymbol{\Phi}_{k-1}+\mathbf{Q}_{k-1},
$$

where the error covariance matrix $\mathbf{P}_{k}$, defined by

$$
\mathbf{P}_{k} \triangleq \mathrm{E}\left[\left(\mathbf{X}_{k}-\hat{\mathbf{X}}_{k}\right)\left(\mathbf{X}_{k}-\hat{\mathbf{X}}_{k}\right)^{\prime}\right]
$$

is updated by using the relation

$$
\mathbf{P}_{k}=\left(\mathbf{I}_{2}-\mathbf{K}_{k} \overline{\mathbf{H}}_{k}\right) \mathbf{P}_{k}^{-},
$$

where $\mathbf{I}_{2}$ is the $2 \times 2$ identity.

Initial conditions. To execute the above iterations, we need knowledge of the initial conditions for the state estimator, the error covariance matrix, and the predictor estimate. These are given as follows:

$$
\hat{\mathbf{X}}_{0}=\overline{\mathbf{X}}_{0},
$$

where $\overline{\mathbf{X}}_{0}$ is given by Eq. (8),

$$
\mathbf{P}_{0}=\left[\begin{array}{cc}
\sigma_{A_{0}}^{2} & 0 \\
0 & \sigma_{B_{0}}^{2}
\end{array}\right],
$$

and, finally,

$$
\hat{\mathbf{X}}_{1}^{-}=\boldsymbol{\Phi}_{0} \overline{\mathbf{X}}_{0}+\mathbf{M}_{0}^{\prime},
$$

where $\boldsymbol{\Phi}_{0}$ and $\mathbf{M}_{0}$ are given by Eqs. (5) and (7), respectively.

As is the case in all Kalman estimators, the estimate $\hat{\mathbf{X}}_{k}$ is the sum of two terms: the predictor $\hat{\mathbf{X}}_{k}^{-}$, defined by Eq. (14), and a correction of the prediction, given by the second term on the right-hand side of Eq. (13).

We conclude this section by indicating that in many practical cases that we have studied, the temporal readout noise is found to be approximately the same through all the detectors in the FPA and over blocks of frames. Also, we have found that it is quite possible that the range of input irradiance is invariant from block to block (i.e., $T_{k}^{\max }$ and $T_{k}^{\min }$ do not change with $k$ ). Under the above assumptions, the Kalman gain matrix $\mathbf{K}_{k}$, the error covariance $\mathbf{P}_{k}$, and the predictor error covariance $\mathbf{P}_{k}^{-}$ are all independent of $k$ and also common to all detectors. These quantities can therefore be computed off line. In this situation, the on-line computations per detector and per block of frames include the predictor estimate $\hat{\mathbf{X}}_{k}^{-}$and the prediction correction term, which is given by the second term on the right-hand side of Eq. (13). Hence the algebraic operations involved (per detector and per block of frames) are the product of a $2 \times 2$ matrix and a 2 $\times 1$ vector, plus the product of a $2 \times l_{k}$ matrix and an $l_{k} \times 1$ vector.

\section{APPLICATION TO SIMULATED DATA}

In this section, the performance of the proposed technique is studied by using IR image sequences that are corrupted by simulated nonuniformity. For convenience and in all simulations, the mean gain is assumed to be unity and the mean value of the bias is taken as zero. The standard deviation of the temporal noise is considered fixed at unity. With these assumptions, blocks of simulated nonuniformity patterns with high, low, and moderate drift levels between consecutive blocks were generated (corresponding to various values of the Gauss-Markov parameters). Further, different levels of artificial nonuniformity were introduced in the simulated block of frames by varying the variance of the gain and the bias. One hundred trials of each case were generated, and each trial included ten blocks, each containing 3000 frames. The initial estimates of the bias and gain matrices were simply taken as the theoretical means (i.e., we initially assumed uniform initial gain and bias matrices).

Two aspects of the performance are considered: (1) the ability of the Kalman filter to estimate the gain and the bias and (2) the use of these estimates to compensate for nouniformity noise in imagery. In this paper, the nonuniformity compensation is performed by simply subtracting the estimated bias from the data and dividing the outcome by the estimated gain. When such an operation is performed on a frame, we call the frame a corrected frame.

\section{A. Performance Metrics}

To study the performance of the Kalman estimator, we use the mean square error (MSE) for the gain and the bias, averaged over all detectors. More precisely, for the $k$ th block, the MSE for the gain is defined as

$$
\mathrm{MSE}_{A_{k}}=\frac{1}{p m} \sum_{i=1}^{p} \sum_{j=1}^{m}\left(\hat{A}_{k}^{i j}-A_{k}^{i j}\right)^{2},
$$

where $p$ and $m$ are the number of rows and columns, respectively, in the FPA. The bias average error $\mathrm{MSE}_{B_{k}}$, associated with the bias estimate $B_{k}$, is defined similarly to Eq. (25).

The NUC capability, on the other hand, is examined by means of three metrics that are commonly used in assessing the fixed-pattern noise in images. These metrics are the roughness parameter $\rho$, the root mean square error RMSE, and the correctability parameter $c .^{1,14,19,20}$ These parameters are defined below. For any image $f$, the roughness parameter is defined by ${ }^{14}$

$$
\rho(f) \triangleq \frac{\left\|h_{1} * f\right\|_{1}+\left\|h_{2} * f\right\|_{1}}{\|f\|_{1}} .
$$

where $h_{1}(i, j)=\delta_{i-1, j}-\delta_{i, j} \quad$ and $\quad h_{2}(i, j)=\delta_{i, j-1}$ - $\delta_{i, j}$, respectively, $\delta_{i j}$ is the Kronecker delta, $\|f\|_{1}$ is the 
$l^{1}$ norm of $f$, and $*$ represents discrete convolution. Note that $\rho$ is zero for a uniform image, and it increases with detector-to-detector variation in the image. Moreover, since $\rho$ does not require the knowledge of the true image, it can be used as a measure of NUC in real IR data as well as simulated data. The RMSE and $c$ parameters require the knowledge of the true scene (or irradiance). These two parameters are therefore applied in this paper to simulated data. The RMSE is defined by ${ }^{19}$

$$
\operatorname{RMSE}=\frac{1}{p m}\left[\sum_{i=1}^{p} \sum_{j=1}^{m}\left(\hat{T}^{i j}-T^{i j}\right)^{2}\right]^{1 / 2},
$$

where $T^{i j}$ and $\hat{T}^{i j}$ are, respectively, the true IR signal and its estimate (i.e., the detector readout after NUC, namely, after subtracting the readout bias and dividing by the detector gain). (Note that for convenience we omit the blocktime subscript $k$ and the frame number $n$ from the signal $T^{i j}$ and its estimate.) Moreover, the RMSE can be similarly computed for the raw frame, which can then be compared with the RMSE value for the corrected frame. Finally, the correctability parameter is computed by using simulated flat-field (FF) data (namely, when all detectors see the same IR signal), and it is defined by ${ }^{20}$

$$
c=\left(\frac{\sigma_{\text {total }}^{2}}{\sigma_{V}^{2}}-1\right)^{1 / 2}
$$

where $\sigma_{V}^{2}$ is the variance of the temporal noise, which can be estimated by using the techniques given in Ref. 14 . $\sigma_{\text {total }}^{2}$ is the spatial sample variance, given by

$$
\sigma_{\text {total }}^{2}=\frac{\sum_{i=1}^{p} \sum_{j=1}^{m}\left(Y^{i j}-\bar{Y}\right)^{2}}{p m-1},
$$

and $\bar{Y}$ is the spatial sample mean of the raw frame. Note that $\sigma_{\text {total }}^{2}$ combines the effect of the temporal noise of each detector as well as the spatial noise. A correctability less than unity indicates that the spatial noise is below the level of the temporal noise, which is a highly desirable outcome for any NUC technique. In particular, if $c=0$, the NUC is perfect, i.e., no spatial noise is present.

\section{B. Dependence of the Performance on the Level of Nonuniformity and Drift}

We studied two cases corresponding to situations where the fixed-pattern noise is dominated by either the gain or the bias nonuniformity. The performance in other cases was also studied, and we will provide comments as needed.

Table 1 shows the empirical MSE in the estimates of the gain and the bias for high, low, and moderate levels of drift. (For brevity, we tabulate the results for only the first three blocks.) The standard deviations for the gain and the bias are 0.15 and 5 , respectively, and the number of frames per block, $l_{k}$, is 3000 . It can be seen that the empirical MSE decreases with the decrease in the drift (i.e., as $\alpha$ and $\beta$ increase). This is expected, since when drift is low (i.e., $\alpha$ and $\beta$ are high), the blocks become more correlated and an estimate at $k$ may use more of the information contained in the previous block. Moreover, our calculations show that, as expected, the error covariance matrix $\mathbf{P}_{k}$ increases with the increase in the drift magnitude but is independent of the block index. This is consistent with our observation that the empirical MSE is nearly independent of $k$. Figures 1, 2, and 3 show a frame of the block used as the true image sequence, the same frame including fixed-pattern noise, and the corrected frame, respectively. The parameters $\rho$ and RMSE, computed for the corrected and raw frames, demonstrate a reduction in the nonuniformity by a factor of 3 and 10 , respectively.

Similar results were obtained when the fixed-pattern noise was generated primarily by the bias. For brevity,

\section{Table 1. Empirical Mean Square Error (MSE) for the Gain and Bias Estimates for Low and High Drift Conditions}

\begin{tabular}{ccccc}
\hline \multirow{2}{*}{$\begin{array}{c}\text { Block No. } \\
k\end{array}$} & $\mathrm{MSE}_{A_{k}}$ & $\mathrm{MSE}_{B_{k}}$ & $\mathrm{MSE}_{A_{k}}$ & $\mathrm{MSE}_{B_{k}}$ \\
\cline { 2 - 5 }$(\alpha=\beta=0.95)$ & $(\alpha=\beta=0.1)$ \\
\hline 1 & $7 \times 10^{-4}$ & 0.434 & $10^{-3}$ & 0.985 \\
2 & $7 \times 10^{-4}$ & 0.436 & $10^{-3}$ & 0.989 \\
3 & $7 \times 10^{-4}$ & 0.432 & $10^{-3}$ & 0.969 \\
\hline
\end{tabular}

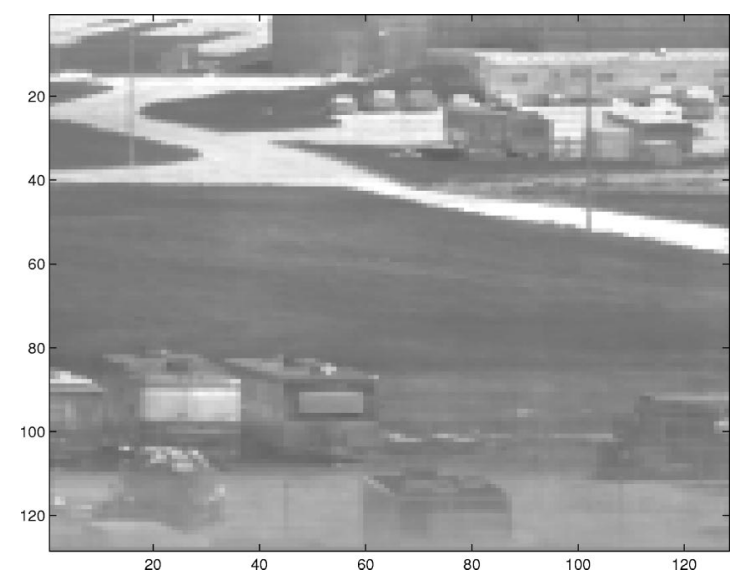

Fig. 1. True 8-bit image from the fifth data set $(k=5)$.

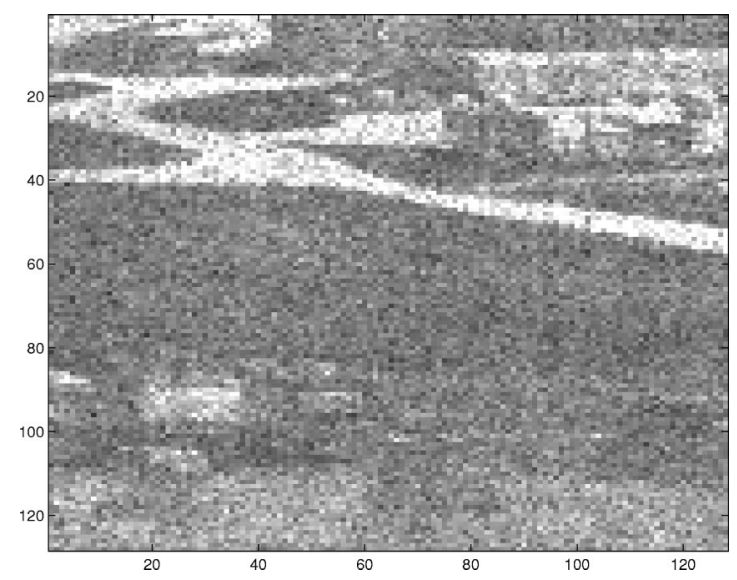

Fig. 2. Image of Fig. 1 corrupted with simulated nonuniformity The nonuniformity is generated with standard deviations for the gain and the offset of 0.15 and 5 , respectively. 


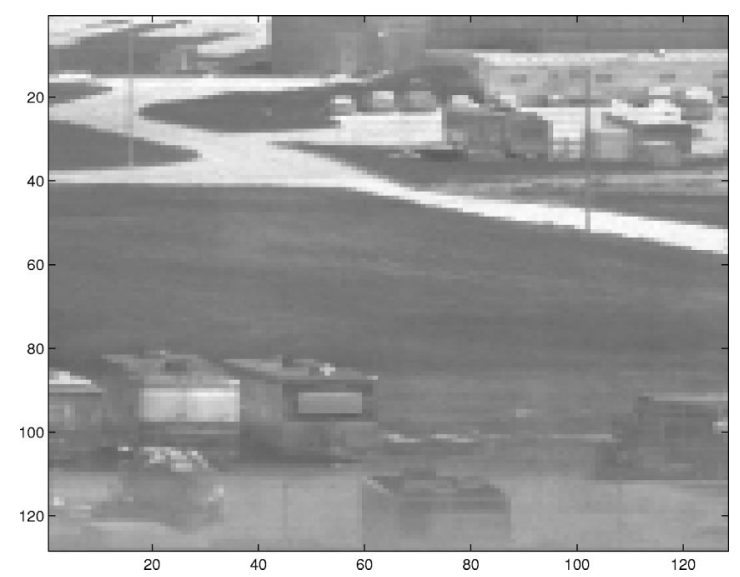

Fig. 3. Corrected version of the image in Fig. 2.

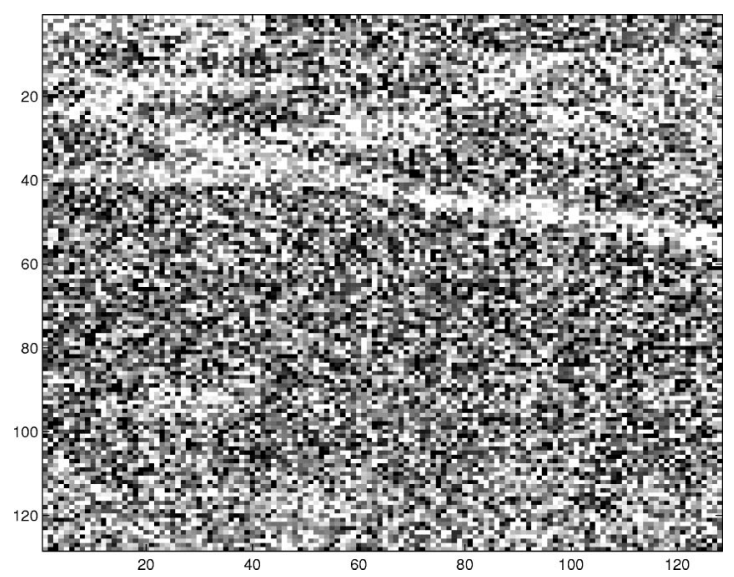

Fig. 4. Image of Fig. 1 with a high level of simulated bias nonuniformity. The gain and offset standard deviations are 0.01 and 100 , respectively.

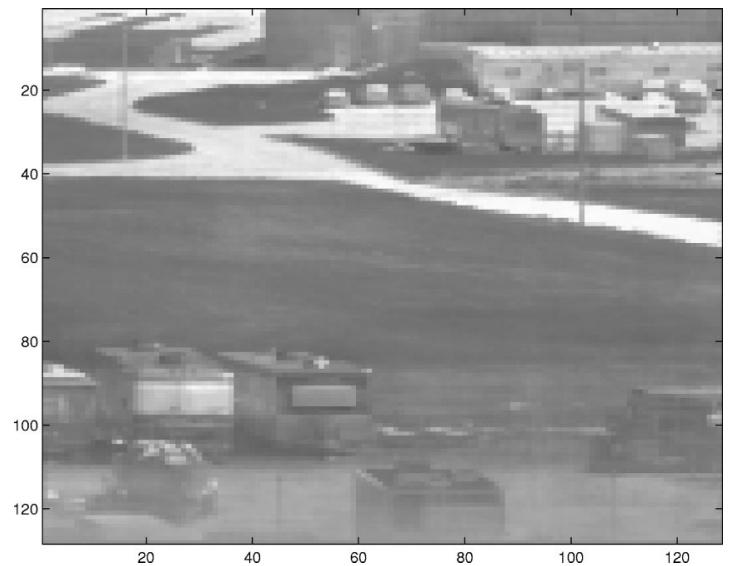

Fig. 5. Corrected version of the image in Fig. 4.

we present only part of the results. Figures 4 and 5 show the frame of Fig. 1 with nonuniformity generated mostly by the bias, and the corresponding corrected frame, respectively. The parameters $\rho$ and RMSE, computed for the corrected and raw frames, reveal a reduction in the nonuniformity by a factor of 12 and 20 , respectively.

C. Dependence of the Performance on the Block Length We tested the Kalman filter by using sequences of simulated data sets with different block lengths. The effect of sampling the frames (as opposed to taking the frames consecutively) was also studied. Sampling frames in time (in each block) generally speeds up the motion and brings about more diversity in the signal levels, which can be beneficial to the performance. Table 2 shows the RMSE performance parameter when the number of sampled frames is varied (the frame sampling was carried out in increments of ten samples). The standard deviations of the gain and the offset are 0.1 and 5 , respectively, and the drift parameters are $\alpha=\beta=0.95$. It was found that when the number of sampled frames was in excess of 250 frames, the RMSE reached its minimum value, which was approximately equal to 5 . We also observed that when the number of frames was less than 100 sampled frames per block, the Gauss-Markov model played a crucial role in improving the quality of the NUC from block to block, in which case the Kalman filter takes more advantage of the information contained in the previous block of frames.

When frames were taken consecutively, on the other hand, the Kalman filter required approximately 1000 consecutive frames before reaching a RMSE close to 5 . This example shows the advantage in efficiency and performance rendered when the frames are sampled. As an example, Figs. 6, 7, and 8 show the corrected images for the case of 50,150 , and 1000 consecutive frames, respectively. Note the artifacts at the bottom of the image in Fig. 6 (and, to a lesser degree, in Fig. 7), which result from the

Table 2. Root Mean Square Error (RMSE) of a Corrected Image for Several Numbers of Sampled Frames per Block

\begin{tabular}{llccc}
\hline \multicolumn{5}{c}{ RMSE } \\
\cline { 2 - 5 }$k$ & $l_{k}=50$ & $l_{k}=100$ & $l_{k}=200$ & $l_{k}=250$ \\
\hline 1 & 22 & 14 & 10 & 5.9 \\
2 & 12.9 & 9.5 & 8.6 & 5.6 \\
3 & 12.7 & 9.2 & 8.6 & 5 \\
4 & 12 & 9 & 8.5 & 5 \\
5 & 12 & 9 & 8.5 & 5 \\
\hline
\end{tabular}

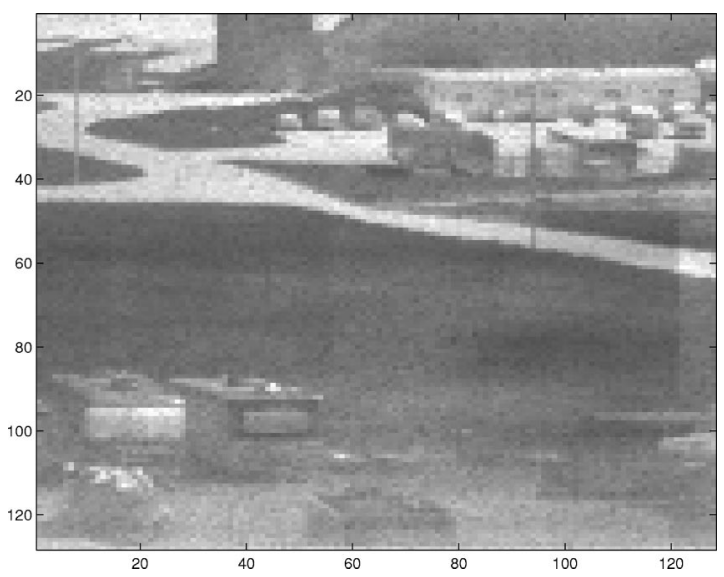

Fig. 6. Nonuniformity correction (NUC) with 50 consecutive frames. Note the artifacts at the bottom of the image, which result from the lack of statistical gray-value diversity within the 50 frames. This lack of diversity violates the constant-range assumption. 


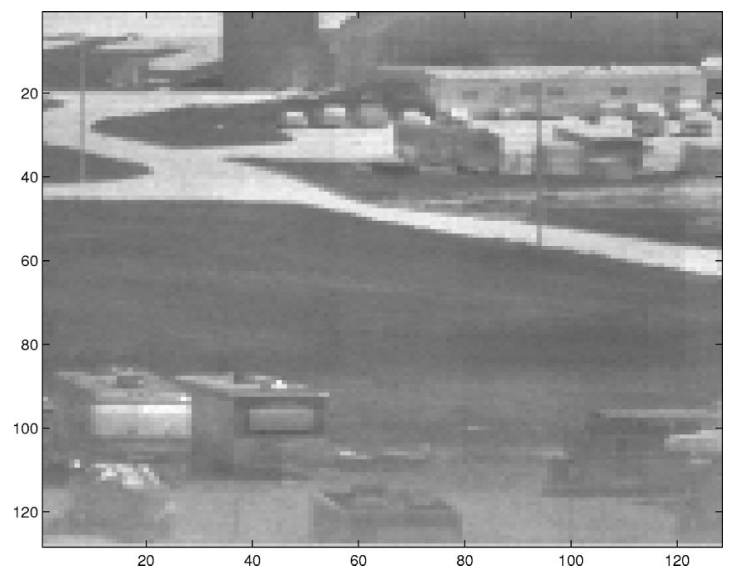

Fig. 7. NUC with 150 consecutive frames. Note the improvement in comparison with Fig. 6; however, some residual artifacts remain, again as a result of a minor violation of the constantrange assumption.

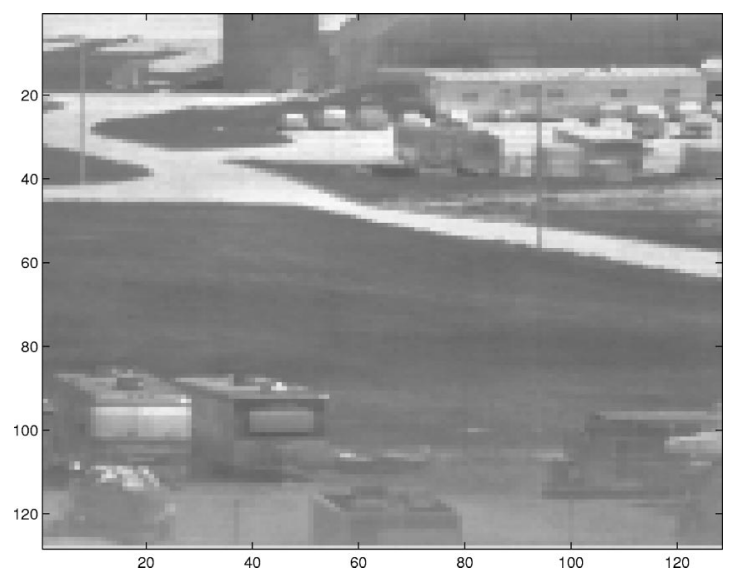

Fig. 8. NUC with 1000 consecutive frames. Note the improvement in comparison with Fig. 7.

Table 3. Correctability Parameter $c$ as a Function of the Temporal-Noise Variance for Six Flat-Field Levels

\begin{tabular}{ccccc}
\hline \multirow{2}{*}{$\begin{array}{c}\text { Flat-Field } \\
\text { Level }\end{array}$} & \multicolumn{4}{c}{ Correctability Parameter $c$} \\
\cline { 2 - 5 } & $\sigma_{\mathbf{V}_{k}}=3$ & $\sigma_{\mathbf{V}_{k}}=1$ & $\sigma_{\mathbf{V}_{k}}=0.5$ & $\sigma_{\mathbf{V}_{k}}=0.2$ \\
\hline 60 & 0.59 & 0.83 & 1.41 & 3.3 \\
80 & 0.57 & 0.74 & 1.2 & 2.7 \\
100 & 0.54 & 0.68 & 1 & 2.34 \\
150 & 0.57 & 0.72 & 1.16 & 2.6 \\
200 & 0.65 & 0.98 & 1.73 & 4.27 \\
240 & 0.75 & 1.26 & 1.63 & 5.85 \\
\hline
\end{tabular}

lack of statistical gray-value diversity within the 50 frames. This lack of diversity is causing the violation of the constant-range assumption.

\section{Dependence of the Performance on the Temporal Noise}

Traditionally, the methodology used to study the dependence of the performance of FPA systems on the temporal noise is based on the procedure proposed by Schulz and Caldwell, ${ }^{20}$ and it involves the correctability parameter $c$.
The procedure involves implementing the NUC method under question with raw FF data and investigating the ability of the NUC technique in reducing the spatial noise to a level below the temporal noise. Here we follow the same procedure and apply the Kalman filter to sequences of 300 frames of simulated noisy (with both temporal and fixed-pattern noise) FF data. Several levels of temporal noise were considered, and each block of frames contained different levels of $\mathrm{FF}$ amplitudes in the gray-scale range 60-240. The results are tabulated in Table 3 for the case of the fifth block $(k=5)$. The standard deviations of the gain and the offset are 0.15 and 5, respectively, and the drift parameters are $\alpha=\beta=0.1$. It can be seen that the proposed technique can reduce the spatial noise to a level slightly below the temporal noise when the standard deviation of the temporal noise is greater than 0.5. Therefore the so-called temporal-noise threshold, which in this case is a number valid for data sets formatted in the 8-bit gray-scale range, is 0.5. Also, note that the correctability parameter is lower in the middle of the FF range (e.g., gray level of 100). The dependence of the performance on the temporal noise was also studied in cases with different levels of nonuniformity noise. In general, the correctability parameter $c$ follows a similar pattern to that in the case discussed here.

\section{E. Sensitivity to Error in the Drift Parameters}

When applying the Kalman estimator to real data, where the Gauss-Markov model parameters $\alpha$ and $\beta$ are unknown, we must assume values for these parameters. To see the sensitivity of the Kalman estimator and the resulting NUC capability on errors in the selection of these drift parameters, we used simulated data, with $\alpha=\beta$ $=0.95$, and examined the performance parameters MSE, RMSE, and $\rho$ as the estimator assumed erroneous parameters. Two cases were considered, comprising sampled and consecutive frames. In the case of sampled frames, sequences of blocks with 300 sampled frames were taken from blocks of 3000 in steps of 10 . It was found that the MSE and the RMSE were not noticeably affected by the error in the parameters $\alpha$ and $\beta$. This insensitivity is due to the fact that the sampling of frames enhances the

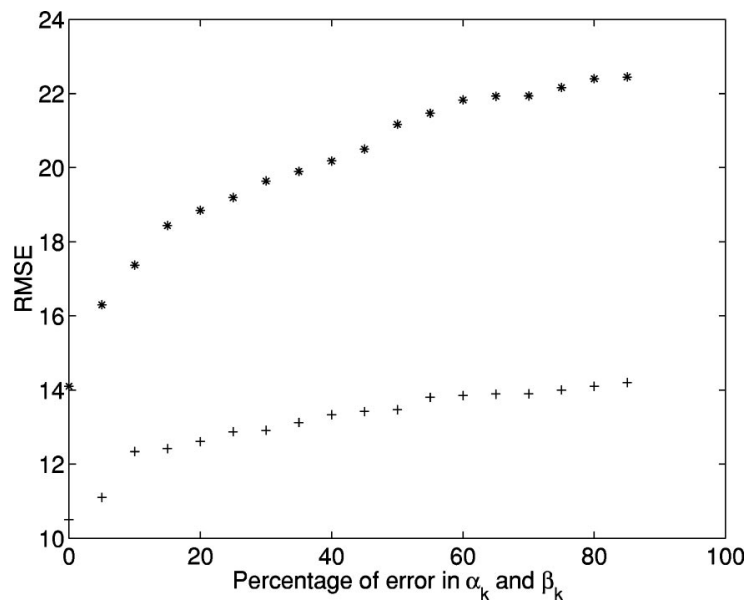

Fig. 9. Root mean square error (RMSE) as a function of the error in the drift parameters $\alpha$ and $\beta$. The symbols* and + correspond to the case with 150 and 300 consecutive frames, respectively. 


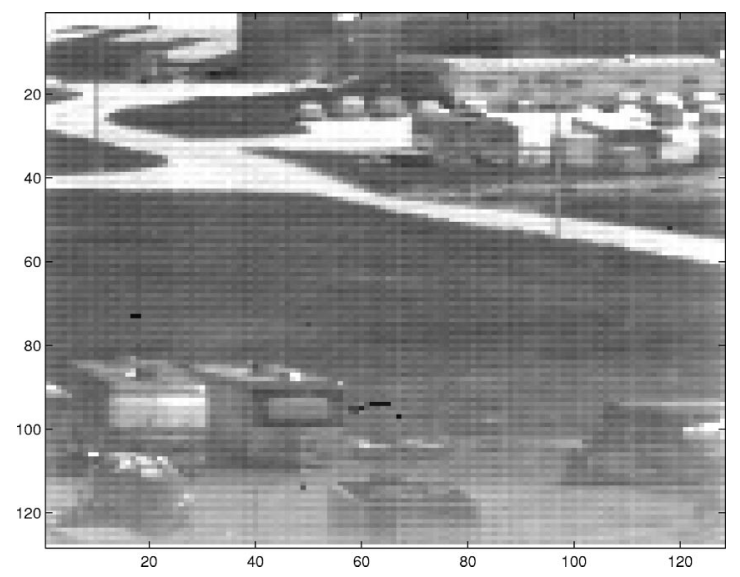

Fig. 10. Raw frame from the second real infrared (IR) data set $(k=2)$.

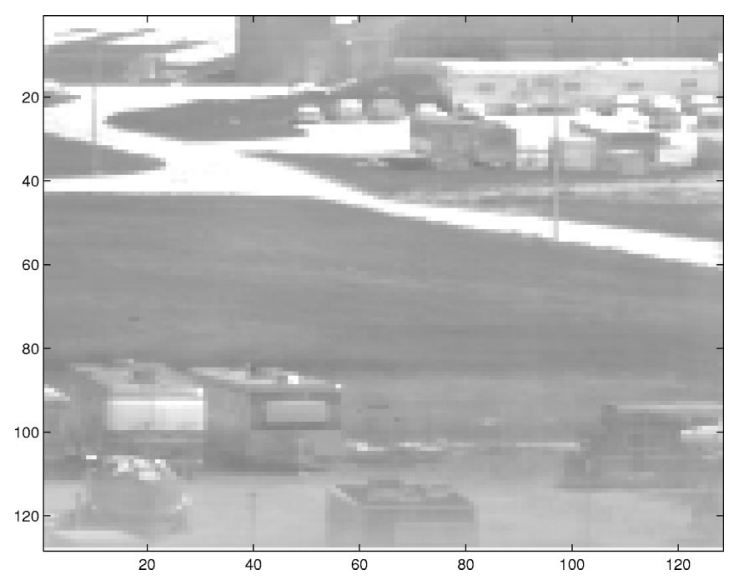

Fig. 11. Corrected version of the image in Fig. 10 using 300 sampled frames. The drift parameters are assumed as $\alpha=\beta$ $=0.95$.

effect of motion, which results in signal diversity. In contrast, when frames were taken consecutively, the performance of the Kalman filter was strongly affected by the errors in the drift parameters unless the number of frames was large (e.g., in excess of 500). For example, when 150 consecutive frames were taken in each block, the RMSE parameter was approximately four times the corresponding value when 300 consecutive frames were used. The dependence of the RMSE on the error percentage in the drift parameters is shown in Fig. 9. The RMSE for a raw frame of the simulated data set for this data set was 35 . Note that even with a high error in the assumed parameters $\alpha$ and $\beta$, the RMSE decreases to the range of 22 with only 150 consecutive frames.

\section{APPLICATION TO REAL INFRARED DATA}

The Kalman filter was applied to two sets of terrestrial IR data. The data sets were collected by using a 128 $\times 128$ InSb FPA camera (Amber model AE-4128) operating in the $3-5-\mu \mathrm{m}$ range. The first set of scenes was collected at 9 a.m., and the second set was acquired at 1 p.m. of the same day. The four-hour time lapse between the two sets of data resulted in observable drift in the respon- sivity of each detector of the FPA. In each set of data, 3000 frames were collected at a rate of 30 frames per second.

For convenience, we assumed that the range of the input irradiance was $[0,255]$ (i.e., $T^{\min }=0$ and $T^{\max }$ $=255$ ). In addition, the initial error covariance matrix $\mathbf{P}_{0}$ and the initial state vector $\overline{\mathbf{X}}_{0}$ were selected within the practical range of the gain and bias values for the above IR FPA camera. ${ }^{14}$ In our calculations, the following set of initial conditions was assumed for all detectors: $\bar{A}_{0}$ $=3.57 \times 10^{3}, \quad \bar{B}_{0}=-5.76 \times 10^{4}, \quad \sigma_{A_{0}}^{2}=1.14 \times 10^{6}$, and $\sigma_{B_{0}}^{2}=4.26 \times 10^{8}$.

Also, since the true drift in the gain and the bias was unknown, the Kalman filter was repeatedly applied while the drift parameters $\alpha$ and $\beta$ were varied in the range 0.05-0.95. As in the case of simulated data, we used the data in two modes: sampled frames and consecutive frames. In the case of sampled frames, a sequence of 300 sampled IR frames (sampled in steps of 10 from the first block of 3000) was used as the input to the Kalman filter at $k=1$ (i.e., $l_{1}=300$ ). With the estimates of the gain and the bias computed at $k=1$, we compensated for the spatial nonuniformity present in the first set of data.

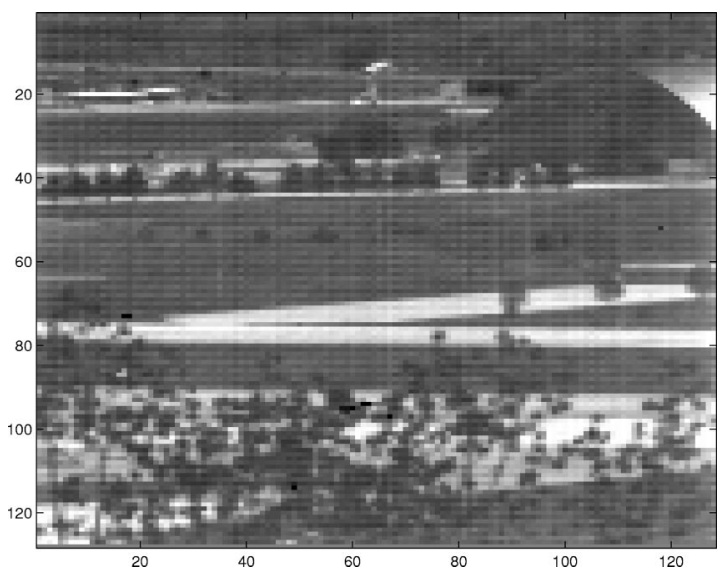

Fig. 12. Example of a raw frame from the second real IR data set $(k=2)$. The dots at the bottom of the image correspond to branches of trees.

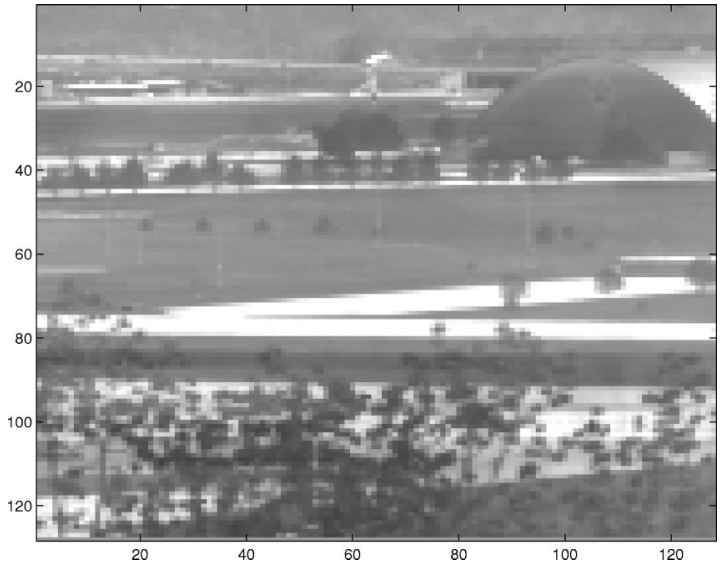

Fig. 13. Corrected version of the image in Fig. 12 using 300 consecutive frames. The drift parameters are assumed as $\alpha=\beta$ $=0.95$, which corresponds to weak drift. 


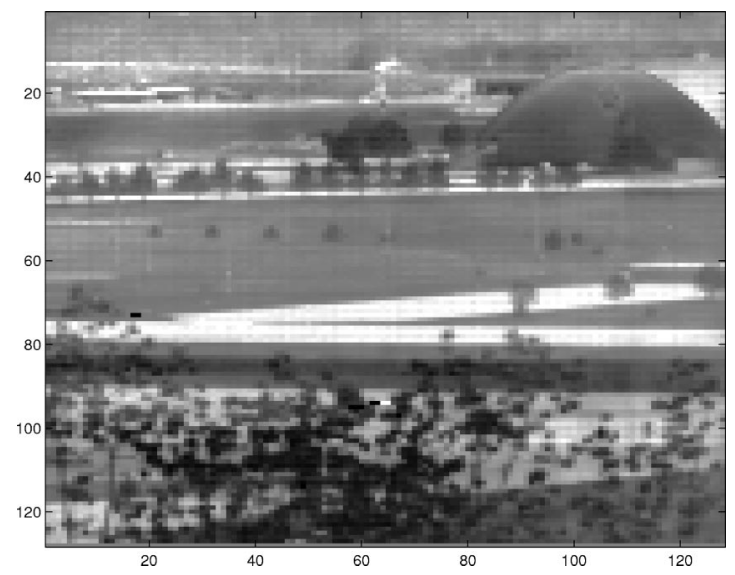

Fig. 14. Corrected version of the image in Fig. 12 using 300 consecutive frames. The drift parameters are assumed as $\alpha=\beta$ $=0.05$, which corresponds to strong drift.

Table 4. Performance Parameter for a Corrected Frame for Different Values of the Drift Parameters $^{a}$

\begin{tabular}{cccccc}
\hline & \multicolumn{2}{c}{ Data Block 1 } & & \multicolumn{2}{c}{ Data Block 2 } \\
\cline { 2 - 3 } \cline { 5 - 6 }$\alpha, \beta$ & $\begin{array}{c}\rho_{s f} \\
\left(\times 10^{-3}\right)\end{array}$ & $\begin{array}{c}\rho_{c f} \\
\left(\times 10^{-3}\right)\end{array}$ & & $\begin{array}{c}\rho_{s f} \\
\left(\times 10^{-3}\right)\end{array}$ & $\begin{array}{c}\rho_{c f} \\
\left(\times 10^{-3}\right)\end{array}$ \\
\hline 0.95 & 1.51 & 1.98 & & 1.81 & 1.98 \\
0.55 & 1.56 & 2.58 & & 1.83 & 3.16 \\
0.05 & 1.58 & 3.2 & & 1.86 & 3.8 \\
\hline
\end{tabular}

${ }^{a}$ Both the sampled frame case $\left(\rho_{s f}\right)$ and the consecutive frame case $\left(\rho_{c f}\right)$ are considered.

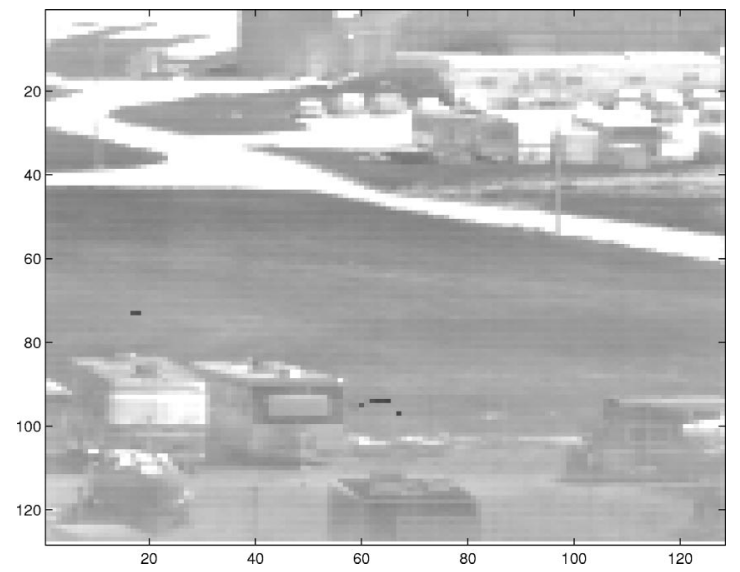

Fig. 15. Corrected version of the image in Fig. 10 using the gain and the bias corresponding to data set 1 . Note that less NUC is achieved in comparison with that in Fig. 11 because of the drift in the gain and the bias. Also note that there is no compensation for the dark spots (dead pixels) that appear in Fig. 10.

The transition to the second data set $(k=2)$ was implemented according to the iterative procedure described in Eqs. (14)-(21).

In the case of sampled frames, it was found that the Kalman filter satisfactorily corrected the nonuniformity in data sets 1 and 2 regardless of the assumed values of the drift parameters. An example is shown in Figs. 10 and 11 for data set 2 , where the selected drift parameters were $\alpha=\beta=0.95$. In contrast to the case of sampled frames, the selection of the drift model parameters plays an important role in the case of consecutive frames. In this case, good NUC was not achieved for values of the drift parameters approximately less than 0.5. In other words, good NUC was achieved only when the estimates of the gain and the bias at $k=2$ appropriately weighed the gain and the bias from the previous block, exploiting the presence of slow drift in the gain and the bias. For example, consider the raw frame of Fig. 12 and compare the good NUC achieved in Fig. 13, with $\alpha=\beta=0.95$ (which is suitable for the weak-drift conditions of the real data collected for this paper), with the poor performance shown in Fig. 14, which corresponds to $\alpha=\beta=0.05$ (which would be suitable for strong-drift cases). This example demonstrates that the Kalman filter is capable of taking advantage of information contained in a previous block of frames and effectively updating this information by using the current block. This observation is also supported through the behavior of performance parameter $\rho$ on the assumed drift parameters, which is shown in Table 4 .

Finally, to emphasize the need for updating the gain and the bias, we attempted to compensate for nonuniformity in data set 2 by using the estimated gain and bias for data set 1 . The raw frame of data set 2 , the corrected image using the gain and the bias for data set 1 , and the corrected image using the updated gain and bias are shown in Figs. 10, 11, and 15, respectively. By comparing Figs. 11 and 15, we see that updating the parameters improves the performance and that the Kalman filter compensates for the dead pixels, as it regards dead pixels as detectors with severe gain nonuniformity. Our calculations have shown that there is an approximate drift of $2.8 \%$ and $3 \%$ for the gain and the bias, respectively, within the fourhour span between data sets 1 and 2 . The simplifying assumption that the driver noise processes $\left[W_{k}^{(1)}, W_{k}^{(2)}\right]^{\prime}$ are white, Gaussian, and mutually uncorrelated seemed to have served us well. Indeed, we have found that the drift in the gain is smaller than the drift in the bias, which is consistent with two-point calibration results.

\section{CONCLUSIONS}

In this paper, we introduced a novel statistical approach for the recursive estimation and correction of gain and bias nonuniformity in focal-plane array sensors. The proposed technique operates on individual blocks of image sequences, which are separated by lengths of time during which drift in the gain and the bias may occur. Through modeling the gain and the bias of each detector by a Gauss-Markov process, the proposed method captures the temporal drift in the gain and the bias in each detector. This memory-inclusive characterization is further exploited in estimating the gain and the bias adaptively as drift occurs. The strength of the drift is captured by the appropriate choice of two drift parameters, which dictate the dependence of the current gain and bias on their respective past values. To achieve the recursive estimation, we derived a Kalman filter, within the confines of the Gauss-Markov model, that estimates the gain and the bias of each detector in each image-sequence block. As a new block of frames arrives, the filter updates the gain 
and the offset accordingly. Once the gain and the offset of each detector are estimated in each block of frames, the fixed-pattern noise is compensated for in the usual way by subtracting from each pixel readout the appropriate detector bias and dividing by the corresponding gain.

The estimation and nonuniformity-compensation capability of the proposed technique was evaluated by employing motion-rich and spatially diverse real IR image sequences as well as image sequences containing simulated fixed-pattern noise. Simulation results indicate that the proposed technique can reduce the fixed-pattern noise to a level that is almost independent of the severity of the noise. Moreover, with the appropriate choice of the drift parameters, the quality of the nonuniformity compensation can be maintained even with high levels of the temporal drift in the detector's parameters. Simulations also show that the proposed technique can reduce the spatial noise to a level below the temporal noise. In cases where the drift is weak and the number of frames per block is low (i.e., in the range of few hundreds of frames per block), good nonuniformity compensation may not be possible unless accurate values for the drift parameters were used to adequately capture the slow drift. This demonstrates how the Gauss-Markov model and the associated Kalman filter provide a mechanism for utilizing past estimates of the detector's parameters in forming improved current estimates.

The approach undertaken in this paper relies on two key assumptions: First, the number of frames used to estimate the gain and the bias in each block of frames must be such that all the pixels are exposed to approximately the same temperature range. This is called the constantrange assumption. Second, the gain and the bias in each detector (and any drift therein) and from detector to detector are uncorrelated. The constant-range assumption turns out to be central to the successful operation of the reported algorithm. Through the use of simulated imagery and real IR data, we showed the consequences that result from violating the constant-range assumption. In the examples considered, such a violation occurred when the number of frames used in each block was small $(<150)$. The result was the appearance of artifacts in the corrected imagery in the form of shaded regions, as distant segments of the array observed different temperature ranges, contrary to the constant-range assumption. However, as the number of frames (per block) was increased, such artifacts quickly disappeared as a result of the presence of motion, which, in turn, guaranteed that all detectors were exposed to the same temperature range. As for the second assumption, the results of applying the algorithm to real IR data showed that this assumption did not seem to be problematic for the examples considered. Albeit, we would generally expect some correlation between the gain and the bias if we consider the nonlinear behavior of the detector output (as a function of the collected photons), which is particularly prominent near saturation. The correlation between the gain and the bias is through their dependence on the random irradiance. If the irradiance is fixed, then it would be safe to assume that the gain and the bias are uncorrelated; however, if the irradiance is allowed to fluctuate in a wide range, causing the detector response to exhibit a nonlinear behavior, then we would start to observe correlation between the gain and the bias as the irradiance varies. Correlation between the biases and the gains of different detector elements is also possible for the same reason, depending on the spatial distribution of the scene. In practice, so long as the detector is operated within its linear range, we would expect the gain and the bias to be uncorrelated. Thus the reason that the assumed absence of correlation between the gain and the bias was not problematic in the examples considered is that the data remained, for the most part, within the linear range of the detector.

Possible extensions of the technique include developing an adaptive method for the estimation of the drift parameters from scene data (i.e., similar to plant estimation in control theory). Future extensions may also accommodate nonlinearities in the detector response. This can be done, for example, by piecewise linearizing the detector response model and applying appropriate versions of the proposed technique to the linear segments.

\section{APPENDIX A}

The standard recursive form of the Kalman estimator $\hat{\mathbf{X}}_{k}$, which is given by Eq. (13), can be found in any of the good references on Kalman filtering (see, for example, Refs. 17 and 18). This recursive form involves the following three quantities: (1) the Kalman gain matrix $\mathbf{K}_{k}$, defined by

$$
\begin{aligned}
\mathbf{K}_{k}= & \mathrm{E}\left[\left(\mathbf{X}_{k}-\hat{\mathbf{X}}_{k}^{-}\right)\left(\mathbf{Y}_{k}-\hat{\mathbf{Y}}_{k}^{-}\right)^{\prime}\right] \\
& \times\left\{\mathrm{E}\left[\left(\mathbf{Y}_{k}-\hat{\mathbf{Y}}_{k}^{-}\right)\left(\mathbf{Y}_{k}-\hat{\mathbf{Y}}_{k}^{-}\right)^{\prime}\right]\right\}^{-1},
\end{aligned}
$$

(2) the predictor estimate $\hat{\mathbf{Y}}_{k}^{-}$of $\mathbf{Y}_{k}$ given $\mathbf{Y}_{1}, \ldots, \mathbf{Y}_{k-1}$, defined by

$$
\hat{\mathbf{Y}}_{k}^{-} \triangleq \mathrm{E}\left[\mathbf{Y}_{k} \mid \mathbf{Y}_{1}, \ldots, \mathbf{Y}_{k-1}\right]
$$

and (3) the predictor estimator $\hat{\mathbf{X}}_{k}^{-}$of $\mathbf{X}_{k}$ given $\mathbf{Y}_{1}, \ldots, \mathbf{Y}_{k-1}$, which is involved in the estimator $\hat{\mathbf{X}}_{k}$ through the standard recursion given by Eq. (14).

What is new in the derivation of the Kalman filter here is the calculation of the Kalman gain $\mathbf{K}_{k}$ and the predictor estimate $\hat{\mathbf{Y}}_{k}^{-}$. We will show these calculations and omit the remaining standard calculations involved in the derivation of the Kalman filter. In deriving the Kalman gain matrix $\mathbf{K}_{k}$ and the predictor estimate $\hat{\mathbf{Y}}_{k}^{-}$, a number of expectations must be computed that involve the random observation matrix $\mathbf{H}_{k}$. Once these expectations are calculated, the remainder of the derivation is straightforward. We first derive the predictor estimate $\hat{\mathbf{Y}}_{k}^{-}$. Using the observation model (10), we can write

$$
\hat{\mathbf{Y}}_{k}^{-}=\mathrm{E}\left[\mathbf{H}_{k} \mathbf{X}_{k} \mid \mathbf{Y}_{1}, \ldots, \mathbf{Y}_{k-1}\right]+\mathrm{E}\left[\mathbf{V}_{k} \mid \mathbf{Y}_{1}, \ldots, \mathbf{Y}_{k-1}\right]
$$

Note that $\mathbf{H}_{k}$ depends on the collected irradiance values $T_{k}(i), i=1, \ldots, l_{k}$, which are independent of the gain and the offset $\mathbf{X}_{k}$. Hence $\mathrm{E}\left[\mathbf{H}_{k} \mathbf{X}_{k} \mid \mathbf{Y}_{1}, \ldots, \mathbf{Y}_{k-1}\right]$ can be expressed as

$$
\begin{aligned}
& \mathrm{E}\left[\mathbf{H}_{k} \mathbf{X}_{k} \mid \mathbf{Y}_{1}, \ldots, \mathbf{Y}_{k-1}\right] \\
&=\mathrm{E}\left[\mathbf{H}_{k} \mid \mathbf{Y}_{1}, \ldots, \mathbf{Y}_{k-1}\right] \mathrm{E}\left[\mathbf{X}_{k} \mid \mathbf{Y}_{1}, \ldots, \mathbf{Y}_{k-1}\right] .
\end{aligned}
$$


Moreover, since $\mathbf{H}_{k}$ is independent of each element of $\mathbf{Y}_{1}, \ldots, \mathbf{Y}_{k-1}, \mathrm{E}\left[\mathbf{H}_{k} \mid \mathbf{Y}_{1}, \ldots, \mathbf{Y}_{k-1}\right]=\mathrm{E}\left[\mathbf{H}_{k}\right]$. Consequently, using the fact that the second term on the right-hand side of Eq. (A2) is zero, we can write the predictor estimate $\hat{\mathbf{Y}}_{k}^{-}$ as

$$
\hat{\mathbf{Y}}_{k}^{-}=\mathrm{E}\left[\mathbf{H}_{k}\right] \mathrm{E}\left[\mathbf{X}_{k} \mid \mathbf{Y}_{1}, \ldots, \mathbf{Y}_{k-1}\right]=\overline{\mathbf{H}}_{k} \hat{\mathbf{X}}_{k}^{-},
$$

where the mean observation matrix $\overline{\mathbf{H}}_{k}$ is given by Eq. (17).

We now show the calculations involved in the derivation of the Kalman gain matrix (A1). First, observe that the matrix $\mathrm{E}\left[\left(\mathbf{Y}_{k}-\hat{\mathbf{Y}}_{k}^{-}\right)\left(\mathbf{Y}_{k}-\hat{\mathbf{Y}}_{k}^{-}\right)^{\prime}\right]$ can be expanded as

$$
\begin{aligned}
& \mathrm{E}\left[\left(\mathbf{Y}_{k}-\hat{\mathbf{Y}}_{k}^{-}\right)\left(\mathbf{Y}_{k}-\hat{\mathbf{Y}}_{k}^{-}\right)^{\prime}\right] \\
& \quad=\overline{\mathbf{H}}_{k} \mathbf{P}_{k}^{-} \overline{\mathbf{H}}_{k}^{\prime}+\mathbf{R}_{k}+\mathrm{E}\left[\mathbf{H}_{k} \mathbf{X}_{k} \mathbf{X}_{k}^{\prime} \mathbf{H}_{k}^{\prime}\right]-\overline{\mathbf{H}}_{k} \mathrm{E}\left[\mathbf{X}_{k} \mathbf{X}_{k}^{\prime}\right] \overline{\mathbf{H}}_{k}^{\prime},
\end{aligned}
$$

where the readout noise covariance matrix $\mathbf{R}_{k}$ and the predictor error covariance matrix $\mathbf{P}_{k}^{-}$are defined by Eqs. (12) and (18), respectively. Then a straightforward (but tedious) calculation shows that

$$
\mathrm{E}\left[\mathbf{H}_{k} \mathbf{X}_{k} \mathbf{X}_{k}^{\prime} \mathbf{H}_{k}^{\prime}\right]-\overline{\mathbf{H}}_{k} \mathrm{E}\left[\mathbf{X}_{k} \mathbf{X}_{k}^{\prime}\right] \overline{\mathbf{H}}_{k}^{\prime}=\sigma_{T}^{2}\left[\sigma_{A_{0}}^{2}+\bar{A}_{0} \mathbf{I}_{\ell_{k}},\right.
$$

where the quantities $\sigma_{T}^{2}, \sigma_{X_{0}^{(1)}}^{2}$, and $\bar{X}_{0}^{(1)}$ are all defined in Section 3. Also, the term $\mathrm{E}\left[\left(\mathbf{X}_{k}-\hat{\mathbf{X}}_{k}^{-}\right)\left(\mathbf{Y}_{k}-\hat{\mathbf{Y}}_{k}^{-}\right)^{\prime}\right]$ can be reduced to

$$
\mathrm{E}\left[\left(\mathbf{X}_{k}-\hat{\mathbf{X}}_{k}^{-}\right)\left(\mathbf{Y}_{k}-\hat{\mathbf{Y}}_{k}^{-}\right)^{\prime}\right]=\mathbf{P}_{k}^{-} \overline{\mathbf{H}}_{k}^{\prime},
$$

and the Kalman gain can be finally expressed as

$$
\mathbf{K}_{k}=\mathbf{P}_{k}^{-} \overline{\mathbf{H}}_{k}^{\prime}\left\{\overline{\mathbf{H}}_{k} \mathbf{P}_{k}^{-} \overline{\mathbf{H}}_{k}^{\prime}+\mathbf{R}_{k}+\sigma_{T}^{2}\left[\sigma_{A_{0}}^{2}+\bar{A}_{0}\right] \mathbf{I}_{\ell_{k}}\right\}^{-1} .
$$

In the above expression, the term $\mathbf{P}_{k}^{-}$is defined by Eq. (18) and can be computed recursively. This recursion can be obtained straightforwardly to yield the recursions given by Eqs. (19) and (21).

\section{ACKNOWLEDGMENTS}

The authors thank Ernest Armstrong and Stephen C. Cain for their valuable suggestions and assistance in this work. This research was supported by the National Science Foundation (CAREER Program MIP-9733308), the U.S. Air Force Research Laboratory (Dayton, Ohio), and the Chilean National Foundation for Science and Technology (FONDECYT).

S. N. Torres's e-mail address is storres@die.udec.cl; M. M. Hayat's e-mail and url addresses are hayat @eece.unm.edu and www.eece.unm.edu.

\section{REFERENCES}

1. G. C. Holst, CCD Arrays, Cameras and Displays (SPIE Optical Engineering Press, Bellingham, Wash., 1996).

2. D. L. Perry and E. L. Dereniak, "Linear theory of nonuni- formity correction in infrared staring sensors," Opt. Eng. 32, 1853-1859 (1993)

3. P. M. Narendra and N. A. Foss, "Shutterless fixed pattern noise correction for infrared imaging arrays," in Technical Issues in Focal Plane Development, W. S. Chan and E. Krikorian, eds., Proc. SPIE 282, 44-51 (1981).

4. P. M. Narendra, "Reference-free nonuniformity compensation for IR imaging arrays," in Smart Sensors II, D. F. Barbe, ed., Proc. SPIE 252, 10-17 (1980).

5. J. G. Harris, "Continuous-time calibration of VLSI sensors for gain and offset variations," in Smart Focal Plane Arrays and Focal Plane Array Testing, M. Wigdor and M. A. Massie, eds., Proc. SPIE 2474, 23-33 (1995).

6. J. G. Harris and Y.-M. Chiang, "Nonuniformity correction using constant average statistics constraint: analog and digital implementations," in Infrared Technology and Applications XXIII, B. F. Andersen and M. Strojnik, eds., Proc. SPIE 3061, 895-905 (1997).

7. Y.-M. Chiang and J. G. Harris, "An analog integrated circuit for continuous-time gain and offset calibration of sensor arrays," J. Analog Integrated Circ. Signal Process. 12, 231-238 (1997).

8. D. A. Scribner, K. A. Sarkay, J. T. Caldfield, M. R. Kruer, G. Katz, and C. J. Gridley, "Nonuniformity correction for staring focal plane arrays using scene-based techniques," in In frared Detectors and Focal Plane Arrays, E. L. Dereniak and R. E. Sampson, eds., Proc. SPIE 1308, 24-233 (1990).

9. D. Scribner, K. Sarkady, M. Kruer, J. Caldfield, J. Hunt, M. Colbert, and M. Descour, "Adaptive nonuniformity correction for IR focal plane arrays using neural networks," in Infrared Sensors: Detectors, Electronics, and Signal Processing, T. S. Jayadev, ed., Proc. SPIE 1541, 100-109 (1991).

10. D. Scribner, K. Sarkady, M. Kruer, J. Caldfield, J. Hunt, M. Colbert, and M. Descour, "Adaptive retina-like preprocessing for imaging detector arrays," in Proceedings of the IEEE International Conference on Neural Networks (Institute of Electrical and Electronics Engineers, New York, 1993), pp. $1955-1960$.

11. W. F. O'Neil, "Experimental verification of dithered scan non-uniformity correction," in Proceedings of the 1996 International Meeting of the Infrared Information Symposium Specialty Group on Passive Sensors (Infrared Information Analysis Center, Ann Arbor, Mich., 1997), Vol. 1, pp. 329 339 .

12. R. C. Hardie, M. M. Hayat, E. E. Armstrong, and B. Yasuda, "Scene-based nonuniformity correction using video sequences and registration," Appl. Opt. 39, 1241-1250 (2000)

13. K. C. Hepfer, S. R. Horman, and B. Horsch, "Method and device for improved IR detection with compensations for individual detector response," U.S. patent 5,276,319 (1994).

14. M. M. Hayat, S. N. Torres, E. E. Armstrong, S. C. Cain, and B. Yasuda, "Statistical algorithm for non-uniformity correction in focal-plane arrays," Appl. Opt. 38, 772-780 (1999).

15. B. M. Ratliff, M. M. Hayat, and R. C. Hardie, "An algebraic algorithm for nonuniformity correction in focal plane arrays," J. Opt. Soc. Am. A 19, 1737-1747 (2002).

16. R. E. Kalman and R. S. Bucy, "New results in linear filtering and prediction: theory," ASME J. Basic Eng. 83, 95107 (1961).

17. H. V. Poor, Introduction to Signal Detection and Estimation (Springer-Verlag, New York, 1988).

18. G. Minkler and J. Minkler, Theory and Applications of Kalman Filtering (Magellan, Palm Bay, Fla., 1993).

19. R. C. Gonzalez and R. E. Woods, Digital Image Processing (Addison-Wesley, New York, 1993).

20. M. Schulz and L. Caldwell, "Nonuniformity correction and correctability of infrared focal plane arrays," Infrared Phys. Technol. 36, 763-777 (1995). 